\title{
La incidencia de la diversidad del directorio en la adopción de prácticas de gobierno corporativo
}

Felipe E. Arenas-Torres', Miguel A. Bustamante-Ubilla ${ }^{2,3^{*}}$ y Roberto M. Campos-Troncoso'

(1) Facultad de Economía y Negocios, Escuela de Auditoría e Ingeniería en Control de Gestión, Centro De Investigación y Estudios Contables, Universidad de Talca, Av. Lircay, Talca, Chile. (Correo-e: farenas@utalca.cl; rcampos@utalca.cl) (2) Facultad de Economía y Negocios, Escuela de Ingeniería Comercial, Universidad de Talca, Av. Lircay, Talca, Chile. . (Correo-e: mabu@utalca.cl)

(3) Sistema de Post Grado, Universidad Católica de Santiago de Guayaquil, Av. Carlos Julio Arosemena, Guayaquil, Ecuador. (Correo-e: miguel.bustamante@cu.ucsg.edu.ec)

Recibido Mar. 30, 2021; Aceptado May. 28, 2021; Versión final Jun. 24, 2021, Publicado Oct. 2021

\section{Resumen}

El objetivo de este estudio es analizar la relación de la diversidad de los integrantes del directorio con la adopción de prácticas de gobierno corporativo de 204 sociedades chilenas durante el período 2015 -2019. Para ello se realizó un análisis descriptivo - correlacional, generando árboles de correlaciones significativas y regresiones que determinan la relación entre variables. Los resultados muestran la existencia de correlaciones significativas entre la diversidad de los directorios, en relación con las variables que clasifican a los accionistas y las que definen la gestión de riesgos. Se aprecia que la diversidad de género incide positiva y significativamente en la adopción de prácticas de gobierno corporativo. Además, la diversidad de nacionalidad incide positiva y significativamente en las prácticas relacionadas con la dimensión accionistas y también con la gestión de riesgos. Se concluye que la diversidad de género y nacionalidad fomentan la adopción de buenas prácticas de gobierno corporativo.

\section{The influence of board diversity on the adoption of corporate governance practices}

\begin{abstract}
The main objective of this study is to analyze the relationship between member diversity in boards of directors and the adoption of corporate governance practices in 204 Chilean companies during the period 2015-2019. A descriptive-correlational analysis is performed, generating trees of significant correlations and regressions to assess relationships between variables. The results show the existence of significant correlations between the diversity of the boards of directors and the variables that classify shareholders and define risk management. Gender diversity positively and significantly affects the adoption of corporate governance practices. Nationality diversity positively and significantly affects practices related to shareholders and risk management. It is concluded that diversity in both gender and nationality encourages the adoption of good corporate governance practices.
\end{abstract}




\section{INTRODUCCIÓN}

En Chile, durante la última década se ha analizado la efectividad de los órganos de gobierno corporativo (GC), respecto del control ejercido sobre las sociedades (Moraga y Rossi, 2019) y como ha sido explicado por Isidro y Sobral (2015) respecto de la influencia de las mujeres en los directorios y el valor de la empresa, además de la existencia de techos de cristal en su conformación, confirmando la existencia de debilidades normativas que rijan a los GC de las empresas en Chile (Arenas et al., 2019). El otro tema que ha sido estudiado es el efecto de la presencia de la mujer en los altos cargos directivos sobre el desempeño financiero y el cumplimiento ético de las organizaciones (Isidro y Sobral, 2015). A modo de ejemplo Terjesen et al. (2015) luego de estudiar 67 países, señalan que las mujeres integran en promedio un 10,3\% de los consejos de administración, sin embargo, no todas las variables de diversidad concentran suficientes estudios (Baker et al., 2020). Respecto de la diversidad, se observa que las tasas más bajas se encuentran en Marruecos $0,0 \%$, Japón $0,9 \%$ y Chile $2,4 \%$ y las más altas en Noruega $42 \%$, Suecia $28 \%$, Finlandia $27,2 \%$ y Francia $22 \%$. Por otra parte al estudiar la zona europea, se aprecia la alta efectividad de la diversidad en cargos estratégicos a causa de las Leyes de Cuota (Mateos de Cabo, 2017) ubicándose alrededor del 15\% (Terjesen et al., 2015).

En el contexto Iberoamericano, solo España ha adoptado una política de cuota de género en los Directorios, siendo su cumplimiento de un $9 \%$ siete años después de su implementación (De Cabo et al., 2019). Sin embargo, la diversidad ha sido progresivamente más estudiada sobre todo la relacionada con la diversidad de género (Mendoza et al., 2018), la heterogeneidad de los directorios (Koitiro y Toshiro, 2018) y la incorporación de la etnicidad, nacionalidad, ocupación y edad de sus integrantes (Hillman, 2015). En este sentido Abdullah (2013) señala que el espíritu de diversidad contribuye a la reducción del riesgo de pensamiento grupal limitando el control de las acciones gerenciales.

En general, se afirma que la diversidad incide sobre el desempeño financiero y positivamente sobre el apalancamiento (Koitoro y Toshiro, 2018). Por su parte García et al. (2015) al estudiar el sistema financiero en nueve países desarrollados, concluyen que la diversidad de género en el directorio aumenta el desempeño financiero de los bancos, mientras que la diversidad de nacionalidad lo inhibe. Por último, Mendoza et al. (2018) al estudiar 98 empresas mexicanas, señalan que la integración de la mujer en el directorio conlleva efectos negativos en el endeudamiento y que la diversidad de género no influye significativamente en la rentabilidad.

En contraste con lo indicado Pucheta y Sánchez (2013) detectaron que tanto los resultados univariantes como multivariantes ponen de manifiesto que la presencia de las mujeres en los consejos de administración de las empresas del lbex - 35 de España no se asocian positivamente con la rentabilidad económica, teniendo poca efectividad en la creación de valor, sin embargo, Suárez et al. (2018) evidencian que existe una incidencia positiva entre la diversidad de género de las empresas españolas y la calidad de la información divulgada. Se generan, en consecuencia, beneficios no financieros asociados a las políticas de sostenibilidad a causa de la diversidad en el directorio (Zaid et al., 2020) y que, respecto de la nacionalidad, Cuadrado et al. (2015) concluyen que la diversidad de miembros extranjeros y mujeres impacta positivamente sobre la responsabilidad social de las organizaciones y que la participación de las mujeres en el Consejo de Administración genera un mayor control sobre las acciones gerenciales y la transparencia corporativa que se hace evidente en la mayor participación, afectando positivamente las sesiones de directorio (Zaid et al., 2020).

Los actuales estándares de gobierno corporativo vigentes en Chile corresponden a la Norma de Carácter General №385, la cual es de adopción voluntaria y se reporta bajo el principio de cumplir o no el requisito. Por esta razón las empresas marcan como respuesta la opción "Sí" o "No" según corresponda, ratificando si se adhieran a la política sugerida. Al respecto, la norma específica se entenderá como práctica adoptada cuando las organizaciones cuentan con las políticas aprobadas o cuando estas se encuentran en pleno funcionamiento. La norma se descompone en 4 categorías, 23 principios y 99 prácticas, siendo la primera categoría el "funcionamiento y composición del directorio" con 11 principios y 51 prácticas, la segunda aborda la "relación entre la sociedad, los accionistas y el público en general" con 7 principios y 22 prácticas, la tercera considera la "gestión y control de riesgos" con 4 principios y 22 prácticas, y por último la cuarta categoría "evaluación por parte de un tercero independiente" con 1 principio y 4 prácticas (NCG N³85, 2015).

Sintetizando, el grado de adopción de las prácticas de gobierno corporativo NCG ํ385, en el trienio 20152017 fue bajo (Arenas et al., 2019), alcanzando un grado de adopción promedio de 31,6\% el año 2015 y de $32,7 \%$ el año 2016 (Moraga y Rossi, 2019). Sin embargo, las empresas buscan dar cumplimiento a la norma sin asumir con convicción la conducta (Di Tommaso, 2018) observándose alta concentración de capital y, a causa de ello, presencia de accionistas controladores que disminuyen la capacidad de los directorios para mejorar sus prácticas de gobierno (Zegers y Consiglio, 2013). En consecuencia y sobre la base de los conceptos y evidencias descritos, el presente trabajo analiza la diversidad de los integrantes del directorio de las sociedades anónimas abiertas chilenas, informadas a la Comisión para el Mercado Financiero (CMF) en 
sus reportes oficiales a contar del año 2015 y hasta el año 2019, a fin de determinar la incidencia en la adopción de prácticas de Gobierno Corporativo (GC), planteándose la hipótesis $\mathrm{H}_{0}$ : La diversidad de género, nacionalidad y edad entre los integrantes del directorio, inciden positivamente en la adopción de prácticas de gobierno corporativo establecidas en la Norma de Carácter General, NCG №385.

\section{METODOLOGÍA}

El presente trabajo de tipo descriptivo y correlacional (Hernández-Sampieri y Mendoza, 2018) fue realizado a través del análisis estadístico de regresión de las variables de diversidad respecto de las cuatro categorías de la Norma de Carácter General (NCG) №385 (2015). Se utilizó además la técnica de árboles de correlaciones a fin de representar de manera esquemática las relaciones entre las áreas de gobierno corporativo, diversidad de género, nacionalidad y edad (Apicella et al., 2009; Gladman y Lamb, 2013; Cuadrado et al., 2015).

La recolección de los datos se realizó a través de fuentes documentales y estadísticas (Hernández-Sampieri y Mendoza, 2018), específicamente del banco de datos de la CMF, regulador de las sociedades emisoras de valores de oferta pública y que reportan anualmente a partir del año 2015 sus prácticas de gobierno corporativo y de responsabilidad social. Por otra parte y con el propósito de realizar un análisis fundado y suficientemente documentado, el presente estudio accedió a los registros de la población de sociedades anónimas que corresponde a 204 organizaciones, que entregaron en su conjunto un total de 942 reportes a la CMF durante el período 2015-2019.

Todas las variables fueron consideradas en el análisis de correlación, sin embargo, a partir de las perspectivas de diversidad que se expresan en las variables de género, nacionalidad y edad, se observó la necesidad de generar correlaciones bivariadas a fin de establecer relaciones de alta significación $\left(\rho \leq 0,05^{*} \circ \rho \leq 0,01^{* *}\right.$, precisamente para obtener árboles de correlación relevantes. Por otra parte, las regresiones fueron hechas de acuerdo con la definición del modelo de regresión donde la adopción de prácticas de gobierno corporativo fue la variable dependiente y las independientes corresponden a los diversos tipos de diversidad analizados, todo ello, de acuerdo con las fuentes destacadas en la Tabla 1.

El árbol de correlación resulta ser una opción alternativa al análisis tradicional (Lim et al., 1998; Loh y Shih, 1997) en tanto permite clasificar, discriminar y hasta predecir relaciones entre variables. El árbol resultante es un esquema de representación de relaciones dicotómicas múltiples que pueden producir bifurcaciones relevantes e interpretables. Para su elaboración se ejecutaron cuatro pasos consecutivos. Primero se procedió a determinar las correlaciones de la totalidad de las variables puestas en análisis. Segundo, se identificaron secuencias correlacionales que dieron pie a encadenamientos significativos. Tercero, se verificó que las correlaciones determinadas pudiesen ser explicadas por el marco teórico que sostiene el estudio. Finalmente, sobre la base del marco conceptual utilizado, se procedió a depurar los árboles de modo que pudiesen contener un número interpretable de variables correlacionadas.

La NCG N`385 como se muestra en la tabla 1, identifica cuatro categorías, 23 principios y 99 prácticas, abordando tópicos como el funcionamiento y composición del directorio, la relación entre la sociedad, los accionistas y el público en general, la gestión y control de riesgos, y la evaluación de un tercero independiente (NCG N`385, 2015). Mientras que la NCG N³86 aborda la responsabilidad social y desarrollo sostenible a partir del número de personas que componen el directorio, incluyendo variables de género, nacionalidad y rango de edad.

El grado de adopción por parte de las empresas a la NCG №385 fue determinado de la división de las prácticas adoptadas y número de prácticas totales definidas como variables dependientes. Por su parte, las variables independientes fueron representadas por los ítems de género, nacionalidad y edad, clasificadas de acuerdo con los criterios regulatorios de la NCG №386, definiendo cada uno de los campos como el coeficiente entre la frecuencia de género o nacionalidad o edad, dividido por el total de directores de cada una de las sociedades analizadas. Por último, como variables de control, se definieron el tamaño y la actividad económica, las cuales fueron medidas como variables dummy tomando el valor uno (1) o cero (0) en el caso que la empresa cumpla o no con la clasificación de tamaño y actividad económica respectivamente.

El análisis de datos consistió en determinar las correlaciones entre las variables de gobierno corporativo y diversidad del directorio (Hernández-Sampieri y Mendoza, 2018), para luego medir el grado de incidencia a través de regresiones lineales múltiples. Las variables de estudio se dividieron en dos grupos: 1) Variables dependientes, la adhesión a la NCG N³85 y sus cuatro categorías y 2) Variables Independientes, la diversidad de género, nacionalidad y edad en el consejo de administración (tabla 1). 
Tabla 1: Definición y medición de las variables de estudio

\begin{tabular}{|c|c|c|}
\hline Variables dependientes & Medición & Fuente \\
\hline $\begin{array}{l}\text { Adhesión: } \\
\text { NCG N³85 } \\
\text { Categoría } 1 \\
\text { Categoría } 2 \\
\text { Categoría } 3 \\
\text { Categoría } 4 \\
\end{array}$ & $\begin{array}{l}\text { NCG } N^{\circ} 385=N^{\circ} \text { de prácticas adoptadas } / \mathrm{N}^{\circ} \text { de prácticas totales } \\
\text { Cat. } 1 \text { (se calcula igual en todas las categorías) }=\mathrm{N}^{\circ} \text { de prácticas } \\
\text { adoptadas Cat. } 1\left(^{*}\right) / \mathrm{N}^{\circ} \text { de prácticas totales } \\
\left({ }^{\star}\right) \text { Cambiar por otras variables }\end{array}$ & $\begin{array}{l}\text { Superintendencia de } \\
\text { Valores y Seguros, } \\
\text { (2015) } \\
\text { Zaid et al., (2020) }\end{array}$ \\
\hline Variables Independientes & Medición & Fuente \\
\hline $\begin{array}{l}\text { Diversidad de género } \\
\text { Hombres } \\
\text { Mujeres }\end{array}$ & $\begin{array}{l}\text { Prop. Dir. Hombres }=\mathrm{N}^{\circ} \text { de integrantes hombres del Directorio } / \mathrm{N}^{\circ} \text { total } \\
\text { de integrantes del directorio } \\
\text { Prop. Dir. Mujeres }=\mathrm{N}^{\circ} \text { de integrantes mujeres del Directorio } / \mathrm{N}^{\circ} \text { total de } \\
\text { integrantes del directorio }\end{array}$ & \multirow{3}{*}{$\begin{array}{l}\text { Mateos et al., (2017) } \\
\text { Cuadrado et al., } \\
\text { (2015) } \\
\text { Hillman, (2015) } \\
\\
\text { García et al., (2015) } \\
\text { Mishra y Modi, (2016) } \\
\text { Koitiro y Toshiro, } \\
\text { (2018) } \\
\text { Suárez et al., (2018) }\end{array}$} \\
\hline $\begin{array}{l}\text { Diversidad de Nacionalidad } \\
\text { Chilenos } \\
\text { Extranjeros }\end{array}$ & $\begin{array}{l}\text { Prop. Dir. Chilenos }=\mathrm{N}^{\circ} \text { de integrantes Chilenos del Directorio } / \mathrm{N}^{\circ} \text { total } \\
\text { de integrantes del directorio } \\
\text { Prop. Dir. Extranjeros }=\mathrm{N}^{\circ} \text { de integrantes Extranjeros del Directorio } / \mathrm{N}^{\circ} \\
\text { total de integrantes del directorio }\end{array}$ & \\
\hline $\begin{array}{l}\text { Diversidad de edad } \\
\text { Menores a } 30 \text { años } \\
\text { Entre } 30-40 \text { años } \\
\text { Entre } 41-50 \text { años } \\
\text { Entre } 51-60 \text { años } \\
\text { Entre } 61-70 \text { años } \\
\text { Mayores a } 70 \text { años }\end{array}$ & $\begin{array}{l}\text { Prop. Dir. menores a } 30 \text { años (se cálcula igual en todas las variables de } \\
\text { diversidad de edad) }=\mathrm{N}^{\circ} \text { de integrantes menor a } 30 \text { años }\left({ }^{*}\right) / \mathrm{N}^{\circ} \text { total de } \\
\text { integrantes del directorio } \\
\left({ }^{*}\right) \text { Cambiar por otras variables }\end{array}$ & \\
\hline Variables de control & Medición & Fuente \\
\hline Tamaño & $\begin{array}{l}\text { Tamaño empresa, registran Sí = } 1, \text { No = 0, en el tamaño respectivo: } \\
\text { - Grande } \\
\text { - Mediana } \\
\text { - Pequeña } \\
\text { - Micro }\end{array}$ & \multirow{2}{*}{$\begin{array}{l}\text { Mishra y Modi, (2016) } \\
\text { Price y Sun, (2017) } \\
\text { Stoian y Gilman, } \\
\text { (2017) } \\
\text { Fernández y Sarria, } \\
(2018) \\
\text { Grover et al., (2019) }\end{array}$} \\
\hline Actividad Económica & $\begin{array}{l}\text { Actividad Económica Sí = 1, No = 0, en la actividad económica respectiva: } \\
\text { Actividades inmobiliarias y de alquiler; Agricultura, ganadería y } \\
\text { silvicultura; Comercio al por menor y mayor; Construcción; Explotación de } \\
\text { minas; Hoteles, restaurantes y entretenimiento; Pesca; Servicios sociales } \\
\text { y de salud; Sociedades de inversiones; Suministros de electricidad, gas y } \\
\text { agua; Transporte, almacenamiento y comunicaciones; Industrias } \\
\text { Manufactureras }\end{array}$ & \\
\hline
\end{tabular}

\section{RESULTADOS}

En los apartados que se indican más abajo, se presentan los resultados de los análisis descriptivos de la muestra, luego una síntesis esquematizada en la forma de árboles de correlación de las variables analizadas, posteriormente se presentan los resultados de las regresiones respectivas.

\section{Estadísticos Descriptivos}

Los resultados presentados en tabla 2, muestran que el grado de adopción promedio de la NCG №385 de gobiernos corporativos fue de un $34 \%$ durante el período 2015 - 2019, mientras que la categoría con mayor adhesión fue la Categoría 3 de Gestión y control de riesgos con un 40\% ( $n=942)$.

Tabla 2: Estadísticos descriptivos de las variables de estudio

\begin{tabular}{|l|c|c|c|c|c|c|}
\hline \multicolumn{1}{|c|}{ Variables } & Media \% & Mediana \% & Moda \% & Desviación\% & Mínimo \% & Máximo \% \\
\hline Adhesión prácticas (Dependientes) & & & & & & \\
NCG No385 de GC & 34 & 32 & 26 & 20 & 0 & 94 \\
\hline Categoría 1 & 35 & 29 & 22 & 23 & 0 & 100 \\
Categoría 2 & 30 & 27 & 5 & 22 & 0 & 86 \\
Categoría 3 & 40 & 41 & 0 & 29 & 0 & 95 \\
Categoría 4 & 10 & 0 & 0 & 27 & 0 & 100 \\
\hline Diversidad del Directorio (Independientes & & & & & & \\
Integrantes del Directorio & 7,72 & 7,00 & 7,00 & 3,26 & 3,00 & 35,00 \\
\hline Hombres & 93 & 100 & 100 & 12 & 20 & 100 \\
Mujeres & 7 & 0 & 0 & 12 & 0 & 80 \\
\hline Chilenos & 90 & 100 & 100 & 20 & 0 & 100 \\
Extranjeros & 10 & 0 & 0 & 20 & 0 & 100 \\
\hline Menores a 30 años & 1 & 0 & 0 & 4 & 0 & 64 \\
Entre 30 - 40 años & 7 & 0 & 0 & 12 & 0 & 80 \\
Entre 41 - 50 años & 14 & 11 & 0 & 16 & 0 & 100 \\
Entre 51 - 60 años & 29 & 29 & 0 & 21 & 0 & 100 \\
Entre 61 y 70 años & 32 & 29 & 29 & 20 & 0 & 100 \\
Mayores a 70 años & 17 & 14 & 0 & 21 & 0 & 100 \\
\hline
\end{tabular}


En contraste, la categoría con menor adopción es la Categoría 4 de "Una evaluación por parte de un tercero independiente" con un $10 \%$. En cuanto a las variables independientes, la media de los integrantes del consejo de administración por empresa fue de 7,72, indicador compuesto en un $93 \%$ por directores hombres y en un $7 \%$ por consejeras mujeres. Respecto de la variable proporción directores extranjeros, esta fue de un $10 \%$. Por último, respecto a la edad de los integrantes del consejo de administración, esta se concentra en un rango entre los 51 y 70 años, abarcando el $61 \%$ del total de consejeros.

\section{Análisis de correlación}

En la figura 1, se observa que todas las correlaciones son significativas al 0,01 bilateral $\left(^{* *}\right)$. Además, entre la variable A) Adopción a la NCG № 385 y la variable B) Funcionamiento y composición del directorio, se genera la correlación más significativa calificada como muy alta, reflejando un coeficiente de $0,925^{\star *}$, de lo que se puede inferir que un buen funcionamiento del directorio podría aumentar el grado de adopción a las prácticas de gobierno corporativo que propone la CMF. En segunda instancia, la variable A) Adopción a la NCG №385, también se correlaciona significativamente con las variables $C$ ) Relación entre la sociedad, los accionistas y el público y D) De la gestión y control de riesgos con un $0,815^{* *}$ y $0,807^{* *}$ respectivamente, situación contraria a la relación entre A) Adopción a la NCG N³85" y la variable E) De la evaluación por parte de un tercero que alcanza un $0,495^{* *}$ y se clasifica como una correlación positiva pero moderada. Sintetizando, se esperaría que el funcionamiento del directorio, las relaciones con los accionistas o la gestión de riesgos, así como grandes áreas de gobierno corporativo, juntos o por si solos, influyan significativamente en la adopción de prácticas de gobierno corporativo.

Adicionalmente, en la figura 1, desde la variable B) Adopción a la NCG N³85, se observan correlaciones altas hacia las categorías C) Relación entre la sociedad, los accionistas y el público en general y D) La gestión y control de riesgos, y moderada con la categoría E) La evaluación por parte de un tercero, las cuales ascienden a $0,630^{* *}, 0,578^{* *}$ y $0,469^{* * *}$ respectivamente. Lo anterior es relevante para el análisis, debido a que un buen funcionamiento y adecuada composición del directorio, podría mejorar áreas tan sensibles como el control de riesgos y permear las diferentes unidades de la organización.

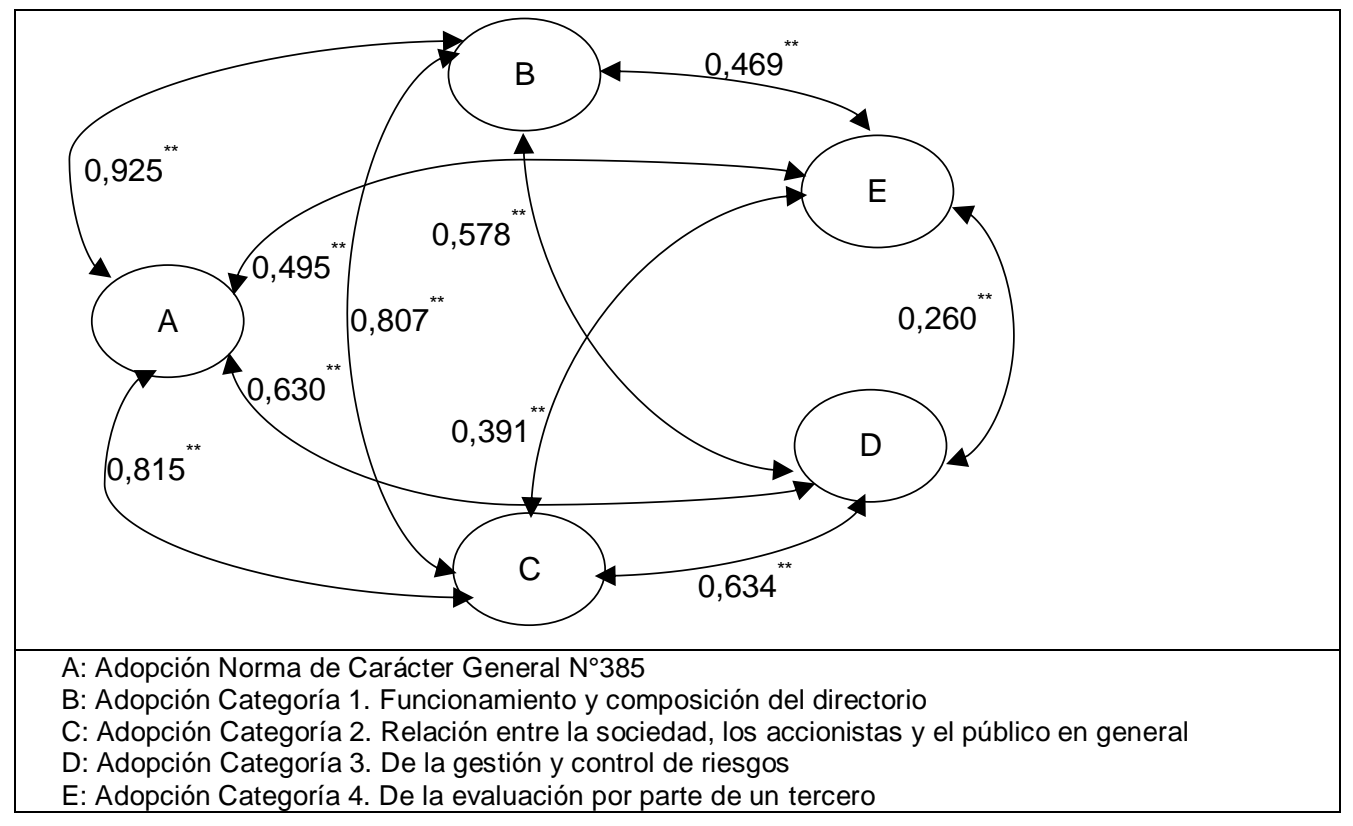

Fig. 1: Árbol de correlaciones de la NCG N³85 de gobierno corporativo

Complementariamente, se observa en la figura 2 que, a partir de la variable G) Proporción de directores mujeres, se establecen correlaciones significativas en el nivel 0,01 y 0,05 bilateral con las variables de edad y género respectivas ( $B, F, J, L$ y $N$ ). Primero, se demuestra la existencia de una correlación positiva, baja y significativa entre la variable $\mathrm{G}$ ) Proporción de directores mujeres y B) Funcionamiento y composición del directorio $\left(0,065^{*}\right)$. Adicionalmente $\mathrm{G}$ ) Proporción de directores mujeres se correlaciona de manera negativa y perfecta con F) Proporción de directores hombres $\left(-1,000^{*}\right)$. También G) Proporción de directores mujeres se correlaciona de manera negativa, significativa y débil con las variables $L$ ) Proporción de directores entre 41-50 años $\left(-0,111^{* *}\right)$ y $\left.\mathrm{N}\right)$ proporción de directores entre $61-70$ años $\left(-0,158^{* *}\right)$. 


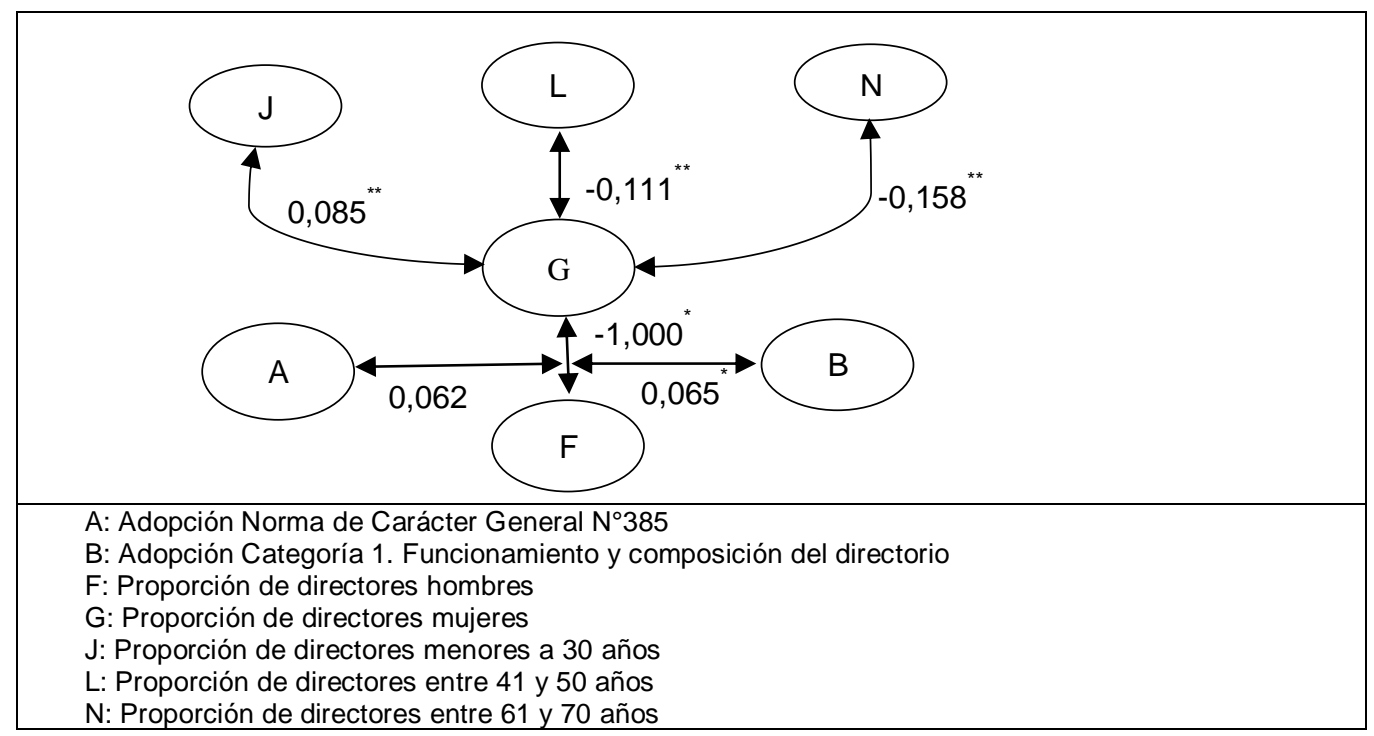

Fig. 2: Árbol de correlaciones significativas a partir de la diversidad de género

Por otra parte G) Proporción de directores mujeres se relaciona con J) Proporción de directores menores a 30 años de manera positiva, significativa y baja $\left(0,085^{* *}\right)$. Finalmente, los datos permiten afirmar que la proporción de directores mujeres $(\mathrm{G})$ se relaciona negativamente con la variables Proporción de directores entre 61 y 70 años $(\mathrm{N})$ y Proporción de directores entre 41 y 50 años $(\mathrm{L})$, mientras que se relaciona en forma positiva con el rango etario medio J) Proporción de directores menores a 30 años. En relación a la diversidad de nacionalidad (figura 3), el árbol de correlaciones considera un mayor número de relaciones significativas en comparación a la figura 2.

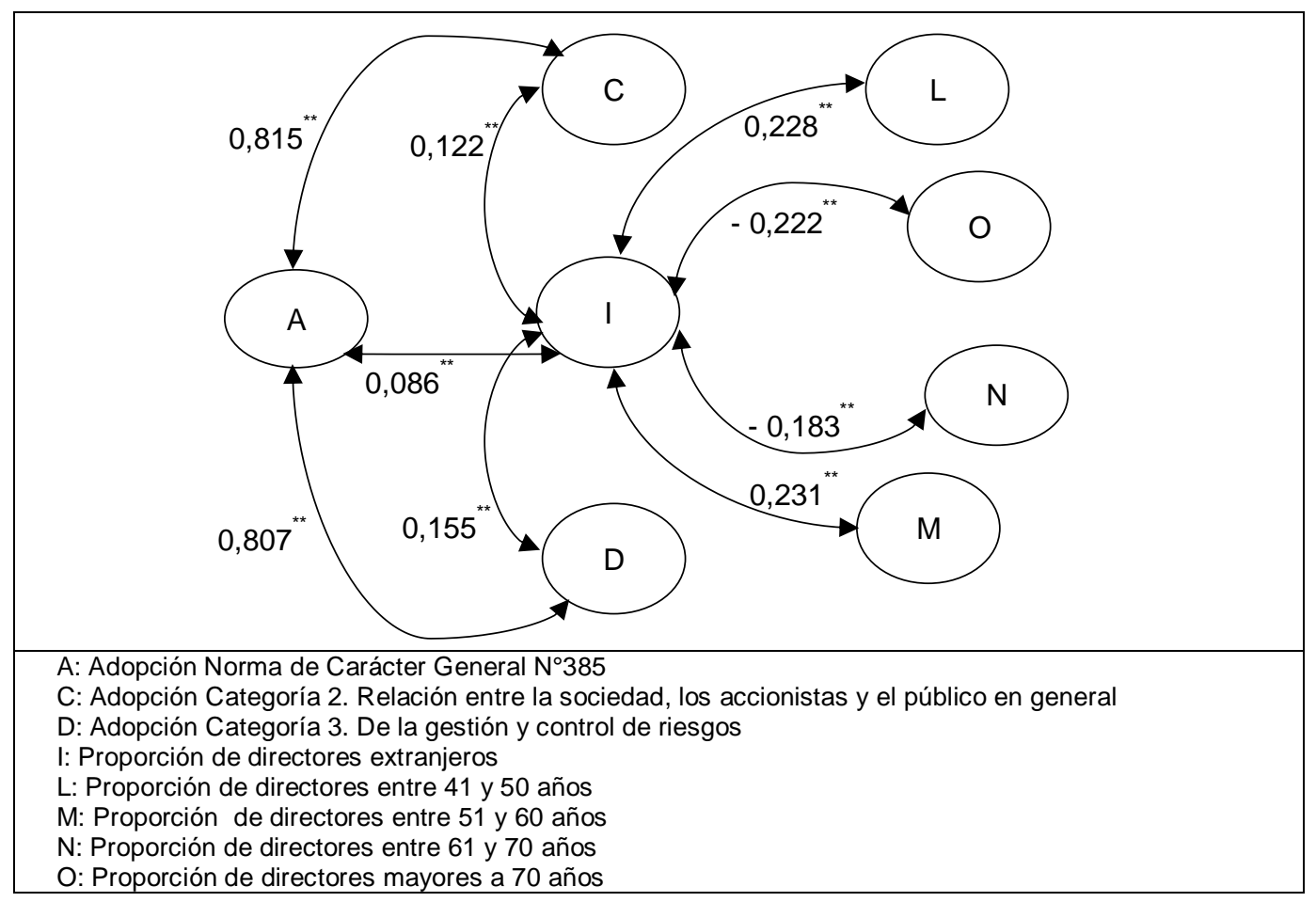

Fig. 3: Árbol de correlaciones significativas a partir de la diversidad de nacionalidad

Primeramente, en cuanto a las variables de edad, I) de Proporción de directores extranjeros se correlaciona positivamente con L) Proporción de directores entre 41 y 50 años $\left(0,228^{* *}\right)$ y M) Proporción de directores entre 51 y 60 años $\left(0,231^{* *}\right)$ abarcando el rango etario de 41 a 60 años, caracterizando impactos bajos. En contraste la variable I) Proporción de directores extranjeros se correlaciona negativamente con N) Proporción de directores entre 61 y 70 años $\left(-0,183^{* *}\right)$ y O) Proporción de directores mayores a 70 años $\left(-0,222^{* *}\right)$. En consecuencia, la variable la Proporción de directores extranjeros (I) se correlaciona en forma directa con los rangos de edad más joven expresados en las variables Proporción de directores entre 41 y 50 años (L) y Proporción de directores entre 51 y 60 años (M) ampliando su efecto a un rango mayor (41 - 60 años) e 
inversa con los rangos de edad más avanzada que se muestran en las variables Proporción de directores entre 61 y 70 años $(\mathrm{N})$ y la Proporción de directores mayores a 70 años $(\mathrm{O})$ ampliando también su rango de efectos sobre las edades (61 hasta 70 años y más).

A partir de la figura 3, la variable I) Proporción de directores extranjeros, registra una correlación positiva muy baja con la variable A) Adopción a la NCG №385, en cambio con la variable C) Relación entre la sociedad, los accionistas y el público en general esta correlación aumenta y se vuelve significativa, al igual que D) De la gestión y control de riesgos con $0,122^{* *}$ y $0,155^{* *}$ respectivamente. Lo anterior pudiera deberse a que los directores extranjeros representan la participación de inversionistas internacionales y por tanto recurren al monitoreo de los accionistas a distancia, lo que establece una relación con los accionistas y el control de riesgos de la empresa, mismas que son reconocidas como áreas fundamentales de adopción de buenas prácticas de gobierno corporativo.

\section{Análisis de regresión}

En la tabla 3 se presentan los resultados de las regresiones que determinan los respectivos índices de incidencia sobre la adhesión de cada una de las variables analizadas destacando en cada caso los coeficientes de significación respectivos.

Tabla 3: Regresión diversidad del directorio y adopción a las prácticas de GC según categorías

\begin{tabular}{|l|c|c|c|c|c|}
\hline \multicolumn{1}{|c|}{ Variables } & NCG No385 & Categoría 1 & Categoría 2 & Categoría 3 & Categoría 4. \\
\hline Coeficientes y correlaciones & Modelo 1 & Modelo 2 & Modelo 3 & Modelo 4 & Modelo 5 \\
\hline Directores Mujeres & $0,115^{*}$ & $0,136^{* *}$ & $0,142^{*}$ & 0,057 & 0,026 \\
Correlación & 0,062 & $0,065^{*}$ & 0,057 & 0,029 & 0,044 \\
\hline Directores Extranjeros & 0,045 & $-0,016$ & $0,091^{* *}$ & $0,141^{* *}$ & 0,028 \\
Correlación & $0,086^{* *}$ & 0,010 & $0,122^{* *}$ & $0,155^{* *}$ & 0,058 \\
\hline Directores menores a 30 años & $-0,252$ & $-0,224$ & $-0,226$ & $-0,401$ & 0,068 \\
Correlación & $-0,055$ & $-0,018$ & $-0,054$ & $-0,099^{* *}$ & $-0,011$ \\
\hline Directores entre 30 y 40 años & $-0,188$ & $-0,400$ & $-0,047$ & 0,141 & $-0,066$ \\
Correlación & $-0,142^{* *}$ & $-0,127^{* *}$ & $-0,110^{* *}$ & $-0,118^{* *}$ & $-0,131^{* *}$ \\
\hline Directores entre 41 y 50 años & $-0,295$ & $-0,511$ & $-0,166$ & 0,041 & $-0,087$ \\
Correlación & $-0,097^{* *}$ & $-0,109^{* *}$ & $-0,073$ & $-0,042$ & $-0,088^{* *}$ \\
\hline Directores entre 51 y 60 años & $-0,107$ & $-0,269$ & $-0,027$ & 0,146 & 0,123 \\
Correlación & $0,155^{* *}$ & $0,123^{* *}$ & $0,137^{* *}$ & $0,158^{* *}$ & 0,055 \\
\hline Directores entre 61 y 70 años & $-0,182$ & $-0,390$ & $-0,064$ & 0,127 & 0,113 \\
Correlación & $-0,043$ & $-0,049$ & $-0,018$ & $-0,032$ & $-0,004$ \\
\hline Directores mayores a 70 años & $-0,089$ & $-0,266$ & $-0,014$ & 0,190 & 0,223 \\
Correlación & 0,042 & $0,073^{*}$ & 0,002 & $-0,016$ & $0,088^{* *}$ \\
\hline Constante & $0,554^{*}$ & $0,820^{* *}$ & 0,307 & 0,264 & 0,119 \\
VC Tamaño & $\mathrm{Si}$ & $\mathrm{Si}$ & $\mathrm{No}$ & $\mathrm{No}$ & $\mathrm{No}$ \\
VC Sector Económico & $\mathrm{Si}$ & $\mathrm{No}$ & $\mathrm{Si}$ & $\mathrm{Si}$ & $\mathrm{No}$ \\
$\mathrm{R}^{2}$ Ajustado & 0,224 & 0,135 & 0,207 & 0,311 & 0,104 \\
\hline Observaciones & 942 & 942 & 942 & 942 & 942 \\
\hline
\end{tabular}

En general se aprecia que efectivamente existen relaciones diversas y significativas $\left(\rho \leq 0,05^{*}\right.$ y $\left.\rho \leq 0,01^{* *}\right)$ entre las variables analizadas, destacando las que se generan entre la proporción de directores extranjeros como variable independiente y la adhesión a la NCG a la NCG №385, adhesión a Cat. 2 Relación entre la sociedad, los accionistas y el público en general y adhesión a Cat. 3 La gestión y control de riesgos con coeficientes de correlación de $0,086^{* *}, 0122^{* *}$ y $0,155^{* *}$ respectivamente. En este mismo análisis, en relación a la edad, destacan los coeficientes de correlación negativos y significativos de correspondencia baja entre la proporción directores entre 30 y 40 años, versus las correlaciones de la NCG №385, y las categorías 1, 2, 3 y 4 con coeficientes de $-0,142^{* *},-0,127^{* *},-0,110^{* *},-0,118^{* *}$ y $-0,131^{* *}$.

Por el contrario, el tramo etario de proporción directores entre 51 y 60 años, presento coeficientes de correlación positivos y significativos de correspondencia baja con la adopción de la NCG No385, y las categorías 1,2 y 3 , las cuales poseen correlaciones de $0,155^{* *}, 0,123^{* *}, 0,137^{* *}$ y $0,158^{* *}$. Al respecto, los tres grandes resultados presentados son relevantes, debido a que los directores de nacionalidad extranjera, junto al grupo etario entre 51 y 60 años son los que pudieran traer un mayor grado de avance en la adopción de prácticas de gobierno corporativo, por el contrario, el grupo entre 30 y 40 años se relaciona de manera inversa.

Detallando el análisis, la proporción directores mujeres posee una incidencia positiva y significativa $\left(\rho \leq 0,05^{*}\right.$; $\rho \leq 0,01^{* *}$ ), de carácter bajo con la variable adopción a la NCG №385 de gobiernos corporativos, con la variable categoría 1 de Funcionamiento y composición del directorio y categoría 2 Relación entre la sociedad, los accionistas y el público en general, alcanzando coeficientes de $0,115^{*}, 0,136^{* *}$ y $0,142^{*}$ respectivamente. 
En relación a la proporción de directores extranjeros, también existe significancia estadística a un nivel de $\rho$ $\leq 0,01^{* *}$, de carácter positivo y bajo, sobre las categorías 3 de La gestión y control de riesgos y categoría 4 de La evaluación por parte de un tercero independiente, mostrando incidencias de $0,091^{* *}$ y $0,141^{* *}$ respectivamente.

Respecto a las proporciones por grupos etarios, se observan coeficientes de incidencia variados, los cuales no muestran incidencia significativa a un nivel de $(\rho \leq 0,05)$ entre las variables dependientes de los modelos 1 al 5 , sin embargo, las correlaciones entre las variables, específicamente en los rangos etarios de directores entre 30 a 40 años y 51 a 60 años son leves pero significativas $(\rho \leq 0,05)$. Por otra parte, al interpretar los mínimos cuadrados ordinarios de los modelos 1 al 5 , se observa que los valores de error de predicción en las variables dependientes son bajos $(0,224,0,135,0,207,0,311$ y 0,104$)$, por tanto, la fiabilidad de las regresiones se encuentran condicionadas al coeficiente de determinación de cada uno de los modelos. Por último, en cada una de las regresiones las variables independientes proporción directores hombres y proporción de directores chilenos, fueron excluidas del análisis, debido a que estas variables son complemento de la proporción de directores hombres y directores extranjeros, confirmando que la variable consejos de administración de directores hombres y chilenos, muestran alta concentración de 0,93 y 0,90 respectivamente.

\section{DISCUSIÓN}

Sin perjuicio de los costos o beneficios de la regulación internacional a los gobiernos corporativos (Di Tommaso, 2018), en este trabajo se observa que existe una baja diversidad de los integrantes del directorio en relación a las variables proporción directores mujeres (De Cabo et al., 2019), extranjeros y grupos etarios (menores a 50 y mayores a 70 años) de un 0,07, 0,10 y 0,40, respectivamente, destacando que este último valor excluye la concentración de directores del grupo etario entre 51 y 70 años. En relación a la diversidad de género, Apicella et al. (2009) identifican que la gestión del riesgo financiero es dominado principalmente por los hombres a lo que se suma lo investigado por Gladman y Lamb (2013) quienes señalan que la participación femenina en las sociedades anónimas abiertas chilenas el 2012 alcanza ya un 3\%, reflejando una evolución que se manifiesta en una leve disminución en la brecha de género en cargos directivos. Respecto a la diversidad de nacionalidad y edad, no existen estudios relevantes para el mercado chileno, sin embargo, Cuadrado et al. (2015) determinaron que en países europeos y norteamericanos los consejeros extranjeros representan en promedio un 16\% del consejo de administración.

En relación a los descriptivos de la NCG N³85, el promedio de adopción en el período 2015 - 2019 es de un $34 \%$, resultados similares a los obtenidos por Arenas et al. (2019) quienes cuantificaron el grado de adopción para el 2015 en un $28,35 \%$, 2016 en un 32,59\% y 2017 en un 34,85\% y lo clasifican como bajo e incipiente, en consecuencia, el avance es marginal luego de dos años de adhesión a las practicas de gobierno corporativo. Sin embargo, se evidencia que los resultados de la adopción de prácticas de la NCG Nº385 se correlaciona fuertemente con la categoría funcionamiento y composición del directorio en un $92,5 \%$, un poco más débil con la categoría relación con las accionsitas en un $81,5 \%$, moderadamente con la categoría gestión y control de riesgos con un $80,7 \%$, y por último incipientemente con la categoría evaluación por parte de un tercero independiente con un 49,5\% respectivamente, siendo todas significativas en el nivel 0,01 biltareal.

Respecto a la incidencia de la diversidad del directorio en la adopción de prácticas de gobierno corporativo, los resultados del estudio son dispares, por ejemplo, las incidencias significativas se materializan respecto de las variable proporción directores mujeres en los modelos 1 Adhesión a la NGC N³85, modelo 2 Adhesión categoría 1" y modelo 3 Adhesión categoría 2" con factores ascendentes a $0,115^{*}, 0,136^{* *}$ y $0,142^{*}$. Mientras que la variable proporción directores extranjeros incide significativamente en los modelos 3 Adhesión categoría 2" y modelo 4 Adhesión categoría 3" con coeficientes de $0,091^{* *}$ y $0,141^{* *}$.

En línea con el punto anterior Cuadrado et al. (2015) identificaron resultados similares a los de este estudio, concluyendo que la diversidad introducida en el directorio a través de miembros extranjeros y mujeres impacta positivamente en el comportamiento socialmente responsable de las organizaciones, siendo la significancia en ambos casos de 0,01, en contraste, Briano y Saavedra (2015) concluyen que la participación de la mujer en el consejo de administración de empresas latinoamericanas no incide significativamente en el nivel de transparencia del gobierno corporativo, sin embargo, Beji et al. (2020) al estudiar las empresas que componen el principal indicador bursátil de Francia, detectaron que la diversidad de género en el Directorio, se asocia positivamente con la dimensión de gobierno corporativo y derechos humanos, señalando que la diversidad de género, nacionalidad y educacional de los miembros del directorio, el involucramiento de directores independientes, mejora la gobernanza y RSE de las sociedades. De igual forma, al estudiar durante el período 2013 a 2018, la evidencia empírica sobre la conexión entre la diversidad de la junta y la adopción de prácticas de sostenibilidad corporativa Zaid et al. (2020) señalan que la adopción de prácticas corporativas relacionadas 
a la sustentabilidad se ven afectadas de manera positiva aunque no significativa por la diversidad de nacionalidad y género en el directorio.

En general, Baker et al. (2020) al analizar 579 estudios de la base de datos ISI actual Web of Science (WOS) para identificar la actividad de investigación relacionada a la diversidad entre 1999 y principios de 2019, detectaron que existe una concentración de investigaciones sobre la diversidad de género en la junta directiva, existiendo relativamente menos investigaciones ligadas a la diversidad de edad, nacionalidad, origen étnico, antecedentes profesionales y la cognición, respecto de cuyas variables este trabajo contribuye con algunos hallazgos, respecto de la edad y nacionalidad de los directivos. Complementariamente, diferentes investigaciones han relacionado la diversidad del directorio (De Cabo et al., 2019) con el funcionamiento del gobierno corporativo y el desempeño financiero de las empresas (Isidro y Sobral, 2015), sin embargo en el caso de Chile, la adopción de prácticas de gobierno corporativo no se relacionan con el grado de insolvencia (Moraga y Rossi, 2019). Por el contrario, a nivel internacional, García et al. (2015) tras un estudio a 159 bancos en 9 países desarrollados, señalan que existe evidencia alentadora puesto que se observaron relaciones positivas entre las variables de diversidad de género y nacionalidad con el desempeño financiero de los bancos.

\section{CONCLUSIONES}

Sobre la base de los hallazgos, resultados, análisis y discusión realizados se puede concluir que la incorporación del concepto de diversidad de género, nacionalidad y edad en los directorios de empresas chilenas es claramente insuficiente, debido a la alta homogeneidad de sus integrantes, permitiendo crear un perfil del director promedio chileno, el cuál principalmente es de género masculino, de nacionalidad chilena y que alcanza un rango etario entre elevado. En consecuencia, después de cinco años de vigencia de la actual normativa la adopción de las prácticas de gobierno corporativo y la adhesión a la NCG N³85 es incipiente, no se visualiza en el mediano plazo cambios significativos en la adopción de las prácticas y tampoco en materia de composición y funcionamiento de los directorios y de gestión de riesgos.

Se ratifica la existencia de una relación positiva (directa) y baja entre la diversidad de género y la adopción a la NCG N`385, lo mismo ocurre con la diversidad de nacionalidad, sin embargo, esta última, se correlaciona significativamente con las categorías relación entre la sociedad, accionistas y gestión y control de riesgos, principalmente a causa de la preocupación de los inversores extranjeros al monitorear sus recursos de manera remota para salvaguardar riesgos potenciales.

Finalmente, se acepta parcialmente $\mathrm{H}_{0}$, debido a que la incidencia de la diversidad de género y la nacionalidad es positiva pero baja, sin embargo el impacto de la diversidad de la edad en la variable dependiente es mayormente negativa (inversa), concluyendo que la diversidad del directorio incide positivamente y en forma directa sobre la adopción de prácticas de gobierno corporativo lo cual promueve la heterogeneidad de los integrantes de los gobiernos corporativos de las sociedades anónimas en Chile.

\section{REFERENCIAS}

Abdullah, S.M., The causes of gender diversity in malaysian large firms, http://dx.doi.org/10.1007/s10997-013-9279-0, Journal of Management Government, 18, 1137-1159 (2014)

Arenas, F., Campos, R., y Santader, R., Adopción de prácticas de gobierno corporativo del mercado chileno en el trienio 2015-2017, http://dx.doi.10.36995/j.visiondefuturo.2019.23.02.008.es, Visión de Futuro, 23(2), $37-55$ (2019)

Apicella, C., Dreber, A., y otros 4 autores, Testosterone and financial risk preferences,

https://doi.org/10.1016/j.evolhumbehav.2008.07.001, Evolution and Human Behavior, 29(6), 384-390 (2009)

Baker, H., Pandey, N., Kumar, S., y Haldar, A., A bibliometric analysis of board diversity: current status, development, and future research directions, https://doi.org/10.1016/j.jbusres.2019.11.025, Journal of Business Research, 108(c) 232$246(2020)$

Beji, R., Yousfi, O., y otros 2 autores, Board diversity and corporate social responsibility: empirical evidence from France, https://doi.org/10.1007/s10551-020-04522-4, Journal of Business Ethics, 1-23 (2020)

Briano, G., y Saavedra, M.L., La composición del consejo de administración y la estructura accionaria como factores explicativos de la transparencia en el gobierno corporativo en latinoamérica: evidencia en empresas cotizadas de Argentina, Brasil, Chile y México, https://doi.org/10.1016/j.estger.2015.02.001, Estudios Gerenciales, 62(136), 275-286 (2015)

Cuadrado, B., García, R., y Martínez, J., Efecto de la composición del consejo de administración en las prácticas de responsabilidad social corporativa, http://dx.doi.org/10.1016/j.rcsar.2014.02.003, Revista de Contabilidad, 18(1), 20-31 (2015) 
De cabo, R., Terjesen, S., y otros 2 autores, Do 'soft law' board gender quotas work? evidence from a natural experiment, https://doi.org/10.1016/j.emj.2019.01.004, European Management Journal, 37(5), 611-624 (2019)

Fernández, J., y Sarria, J., Impact of corporate social responsibility on value creation from a stakeholder perspective, https://doi.org/10.3390/su10062062, Sustainability, 10(6), 2062 (2018)

García, E., García, I., y Martínez, J., Board diversity and its effects on bank performance: an international analysis, http://dx.doi.org/10.1016/j.jbankfin.2014.12.002, Journal of Banking \& Finance, 53, 202-214 (2015)

Gladman, K., y Lamb, M., GMI Ratings' 2013 Women on Boards Survey, GMIRatings (2013)

Di Tommaso, F., What are the costs and benefits of an international corporate governance reregulation? https://doi.org/10.22495/jgr_v7_i3_p, Journal of Governance and Regulation, 7(3), 31-36 (2018)

Grover, P., Kar, A., y llavarasan, V., Impact of corporate social responsibility on reputation - insights from tweets on sustainable development goals by CEOs, https://doi.org/10.1016/j.ijinfomgt.2019.01.009, International Journal of Information Management, 48, 39-52 (2019)

Hernández-Sampieri, R., y Mendoza, C., metodología de la investigación. Las rutas cuantitativa, cualitativa y mixta, Ciudad de México, México: Editorial Mc Graw Hill Education, Año de edición: 2018, ISBN: 978-1-4562-6096-5, (2018)

Hillman, A., Board diversity: beginning to unpeel the onion, https://doi.org/10.1111/corg.12090, Corporate Governance: An International Review, 23(2), 104-107 (2015)

Koitoro, E., y Toshiro, W. Diversity of the board and capital structure, http://dx.doi.org/10.1590/S0034-759020180604, Revista de Administração de Empresas, 58(6), 551-563 (2018)

Lim T.S., Loh W.H., y Shih Y.S., An empirical comparison of decision trees and other classification methods, Technical Report 979, Departments of Statistics, University of Wisconsin, January (1998)

Loh W.Y., y Shih Y.S., Split selection methods for classification trees, Statistica Sinica, 7, 815-840 (1997)

Mateos de Cabo, R., Mujeres y liderazgo empresarial: explorando la brecha de género en la cima, https://doi.org/10.20318/femeris.2017.3758, Revista Femeris, 2(2), 53-80 (2017)

Mendoza, D., Briano, G., y Saavedra, M.L., Diversidad de género en posiciones estratégicas y el nivel de endeudamiento: evicencia en empresas cotizadas mexicanas, http://dx.doi.org/10.21919/remef.v13i4.343, Revista Mexicana de Economía y Finanzas Nueva Época, 13(4), 631-654 (2018)

Mishra, S., y Modi, S., Corporate social responsibility and shareholder wealth: the role of marketing capability, https://doi.org/10.1509/jm.15.0013, Journal of Marketing, 80(1), 26-46 (2016)

Moraga, H., y Rossi, M., Gobierno corporativo y riesgo de quiebra en las empresas chilenas. http://dx.doi.org/10.3232/GCG.2019.V13.N1.06, Revista Globalización, Competitividad y Gobernabiildad, 13(1), 112-125 (2019)

Isidro, H., y Sobral, M., The effects of women on corporate boards on firm value, financial performance, and ethical and social compliance, https://doi.org/10.1007/s10551-014-2302-9, Journal of Business Ethics, 132(1), 1-19 (2015)

Price, J., y Sun, W., Doing good and doing bad: the impact of corporate social responsability and irresponsability on firm performance, http://dx.doi.org/10.1016/j.jbusres.2017.07.007, Journal of Business Research, 80, 82-97 (2017)

Pucheta, M., y Sánchez, P., Relación entre la diversidad de género y la rentabilidad económica de las empresas del IBEX 35, La Ventana, 4(38), 331-372 (2013)

Stoian, C., y Gilman, M., Corporate social responsability that "pays": a strategic approach to CSR for SMEs, https://doi.org/10.1111/jsbm.12224, Journal of Small Business Management, 55(1), 5-31 (2017)

Suárez, Ó., Álvarez, M., y otros 2 autores, Las atribuciones egoístas en los informes de gestión y diversidad de género en los órganos directivos de la empresa, https://doi.org/10.1080/02102412.2018.1478367, Spanish Journal of Finance and Accounting, 48(1), 87-112 (2018)

NCG N`385, Norma de Carácter General N³85 de Gobiernos Corporativos, 1-22, Santiago, Chile (2015)

Terjesen, S., Aguilera, R., y Lorenz, R., Legislating a woman's seat on the board: institutional factors driving gender quotas for boards of directors, http://dx.doi.org/10.1007/s10551-014-2083-1, Journal of Business Ethics, 128, 233-251 (2015)

Zaid, M., Wang, M., y otros 3 autores, Boardroom nationality and gender diversity: implications for corporate sustainability performance, https://doi.org/10.1016/j.jclepro.2019.119652, Journal of Cleaner Production, 251 (2020) 